\title{
$\beta$-Oxo anilides in Heterocyclic Synthesis: Novel Synthesis of Polyfunctionally Pyridines, Pyrimidines and Benzothiazole Derivatives
}

\author{
A. M. Hussein'1, M. S. A. El-Gaby ${ }^{1}$, F. A. Abu-Shenab ${ }^{1}$, M. A. M. Abdel-Raheim ${ }^{1}$ \\ and M. A. El-Apasery ${ }^{2 *}$ \\ ${ }^{1}$ Department of Chemistry, Faculty of Science, Al Azhar University, Assiut 71524, \\ Egypt. \\ ${ }^{2}$ Dyeing, Printing and Textile Auxiliaries Department, Textile Research Division, \\ National Research Centre, 33 El Buhouth St., Dokki, Cairo, Egypt.
}

\begin{abstract}
CETOACETANILIDE derivative 1 was reacted with aromatic aldehydes 2 to yield $A$ the arylidine derivatives 3 and $6 \mathrm{a}, \mathrm{b}$. The Hantzsch amides 7a,b were prepared by the one-pot cyclization reaction of a mixture of 2 moles of 1 , aqueous ammonia and aromatic aldehydes. Treatment of 1 with ethanol containing equivalent amount of piperidine or morpholine furnished the isolable products $8 \mathrm{a}$ and $8 \mathrm{~b}$. Compound 1 underwent interamolecular heterocyclization on boiling conc. sulfuric acid, afforded 9. Also, the reaction of compound 1 with hydroxylamine hydrochloride in ethanol and sodium acetate afforded the oxime derivative 10. Furthermore, reactions of compound 1 with o-aminothiophenol furnished 11. Reactions of 1 with arylidine derivatives give compounds 13 and 16a-d. Treatment of compound $16 \mathrm{~d}$ with elemental sulfur afforded the thieno[3,4-c]pyridine derivative 18. Treatment of 16 a with hydrazine hydrate in boiling ethanol afforded the pyrazolo[3,4-b]pyridine derivatives 19. Also, compound 16a was reacted with ethylchloroacetate giving 20. Compound 20 was cyclized into the corresponding thieno[2,3-b]-pyridine derivative 21 upon boiling with ethanol containing a few drops of sodium ethoxide solution. Furthermore, compound 1 readily reacted with cyanothioacetamide to yield compound 22. Fusion of compound 1 with malononitrile over melting point without solvent in presence of ammonium acetate or refluxing in ethanolic piperidine afforded the pyridone $23 \mathrm{a}$. Also, the pyridone derivative $23 \mathrm{~b}$ was obtained by reacting compound 1 with cyanoacetamide. The reaction of acetoacetanilide 1 with $\omega$-bromoacetophenones afforded 28a,b. Treatment of 1 with benzoyl and ethoxy carbonyl isothiocyanates afforded the pyrimidine derivatives 30a,b. The reaction of 1 with aminopyrazole gave the pyrazolopyrimidine 33. Coupling of 1 with diazonium salt of compounds $34 \mathrm{a}, \mathrm{b}$ yielding $37 \mathrm{a}, \mathrm{b}$.
\end{abstract}

Keywords: Pyridines, Pyrimidines, Dyes, Pyrazolopyridines, Pyrazolopyrimidines, Triazines.

\section{Introduction}

Benzoheterocycles are potent inhibitors of the anaphylactically induced histamine release from rats peritoneal mast cells (RMC) [1]. Especially benzothiazole derivatives have been widely investigated for therapeutic uses as antiviral [2], antibacterial [3], fungicidal [4], antiallergic [1], anti-inflammatory $[5,6]$, as appetite depressants [7], intermediates for dyes [8], plant protectants [9] and photographic sensitizers [10].

In continuation of our previous interest in the synthesis of variety of heterocycles from readily obtainable inexpensive starting materials [11, 12], we report here on the utility of N-1, 3-benzothiazol2-yl-3-oxobutanamide for the synthesis of some novel heterocycles incorporating a benzothiazole moiety.

\section{$\underline{\text { Results and Discussions }}$}

$\beta$-Oxo anilide derivative 1 was reacted with aromatic aldehydes 2 in basic medium afforded the arylidine derivative 3 as literature product $[13,14]$. While, the reaction proceeded by reactions of aromatic aldehydes with $\beta$-Oxo anilide derivative in glacial acetic acid

\footnotetext{
*Corresponding author: Morsy A. El-Apasery; E-mail: elapaserym@yahoo.com DOI: $10.21608 /$ ejchem.2018.3925.1344

(C)2017 National Information and Documentation Center (NIDOC)
} 
afforded a product with water and acetaldehyde elimination. This was formulated as $6 \mathrm{a}, \mathrm{b}$ on the basis of its compatible spectroscopic data. Thus, the mass spectrum of $6 \mathrm{a}$ revealed a molecular ion peak at $\mathrm{m} / \mathrm{z}=312\left(\mathrm{M}^{-2}\right)$ corresponding to the molecular formula $\mathrm{C}_{16} \mathrm{H}_{11} \mathrm{ClN}_{2} \mathrm{OS}$. The IR spectrum showed one absorption band at $3150 \mathrm{~cm}^{-1}$ for $\mathrm{NH}$ and $1686 \mathrm{~cm}^{-1}$ for one carbonyl group. Also, the ${ }^{1} \mathrm{H}$ NMR spectrum of compound $\mathbf{6 b}$ as an example recorded in $\left(\right.$ DMSO- $\mathrm{d}_{6}$ ) revealed a singlet signal at $\delta 3.82$ ppm corresponding to $\mathrm{OCH}_{3}$ protons, doublet at $\delta$ 6.79-6.84 ppm corresponding to olefinic$\mathrm{CH}, J=10.4 \mathrm{~Hz}, 7.02-7.77$, multiplet at $\delta=7.02$ $-7.77 \mathrm{ppm}$ assigned for $\mathrm{CH}$ aromatic, doublet at $\delta$ 7.97-8.00 ppm corresponding to olefinic$\mathrm{CH}, J=10.4 \mathrm{~Hz}$, and singlet signal at $\delta 3.82$ assigned to $\mathrm{NH}$ and absence of the acetyl proton from the molecule. Compound $\mathbf{6}$ is assumed to be formed by the condensation of aldehydic group with methylene group via loss of water followed by cyclization to give the intermediate $5 \mathrm{a}, \mathrm{b}$. In the presence of $\mathrm{H}^{+}$an elimination of acetaldehyde molecule and opening the ring another one to give N-1,3-benzothiazol-2-yl-3(4-substituted phenyl) acrylamide, (Scheme 1). The Hantzsch amides 7a,b were prepared by the one-pot cyclization reaction of a mixture of two moles of compound 1, aqueous ammonia and aromatic aldehydes $[15,16]$. Compound 7 was confirmed by its analytical data (IR, ${ }^{1} \mathrm{HNMR}$ ) and elemental analysis. Thus, the ${ }^{1} \mathrm{HNMR}$ spectrum of compound $7 \mathrm{a}$ as an example, recorded in DMSO- $\mathrm{d}_{6}$ revealed a singlet signal at $\delta=2.26 \mathrm{ppm}$ assigned to $2 \mathrm{CH}_{3}$, singlet signal at $\delta=5.37$ assigned for pyridine- $\mathrm{H}$, multiplet at $\delta=$ $7.14-7.93 \mathrm{ppm}$ assigned for $\mathrm{CH}$ aromatic, and singlet signals at $\delta 9.05,11.93$ assigned to $3 \mathrm{NH}$ protons, (Scheme 1). Treatment of compound 1 with piperidine in ethanol furnished the isolable product $8 \mathrm{a}$ with water elimination. This structure has been established by elemental analysis and spectral data. It's mass spectrum showed a molecular ion peak at $\mathrm{m} / \mathrm{z}=301\left(\mathrm{M}^{+}\right)$and its ${ }^{1} \mathrm{HNMR}$ revealed an absence of $\mathrm{CH}_{2}$ group and revealed the presence of singlet signal at $\delta=$ $1.52 \mathrm{ppm}$ assigned to $\mathrm{CH}_{3}$, multiplet signals at $\delta$ 2.96-3.54 ppm assigned to $\mathrm{CH}_{2}$ protons, singlet signal at $\delta 5.17 \mathrm{ppm}$ assigned to $(\mathrm{CH}=\mathrm{C})$, and multiplet at $\delta 7.18-7.88 \mathrm{ppm}$ assigned to Ar-H and NH protons. Similarly, when compound 1 was treated with morpholine in ethanol afforded compound 8b, (Scheme 1).
Compound 1 underwent interamolecularheterocyclization on boiling concentrated sulfuric acid, afforded 4-methyl-2H-pyrimido[2,1-b] [1,3]-benzothiazol-2-one 9. Also, the reaction of compound 1 with hydroxylamine hydrochloride in ethanol and sodium acetate afforded the oxime derivative (10) based on its elemental analysis and analytical data IR, ${ }^{1} \mathrm{HNMR}$. Furthermore, reactions of compound 1 with o-aminothiophenol in ethanolic triethylamine furnished N-1,3-benzothiazol-2-yl3-methyl-4H-1,4-benzothiazine-2-carboxamide 11, [17-19], (Scheme 2). Reactions of compound 1 with arylidinemalononitrile 12 in ethanolic piperidine may form either 13 or 14. Structure 13 was preferred for the reaction product on the basis of its elemental and spectroscopic data. However, the IR spectrum of compound 13a as an example revealed the presence of an $-\mathrm{NH}_{2}$ group at $v_{\max }=$ 3430 and $3341 \mathrm{~cm}^{-1}$ and the absence of $\mathrm{OH}$ group absorption band. The ${ }^{1}$ HNMR spectrum showed a singlet signal at $\delta=5.71(\mathrm{ppm})$ for $4 \mathrm{H}-$ pyrane whereas structure 14 would be expected to show $\mathrm{OH}$ protons at higher field, (Scheme 2).

$\begin{array}{cccc}\text { The pyridinethiones } & 16 & \text { were } \\ \text { obtained in quantitative yield } & \text { from }\end{array}$ the reaction of acetoacetanilide 1 with arylidinecyanothioacetamide $15 \mathrm{a}-\mathrm{c}$ in ethanolic piperidine rather than the isomeric structure 17. Compound 16 was confirmed based on the spectroscopic data. ${ }^{1}$ HNMR spectrum of compound 16a as an example showed the presence of a singlet signals at $=12.77,14.56 \mathrm{ppm}$ assigned to $(2 \mathrm{NH})$ groups and absence of $4 \mathrm{H}$ thiopyrane would be expected at field $\delta 4-5 \mathrm{ppm}$. Similarly the reaction of ylidinecyanothioacetamide $15 \mathrm{~d}$ (prepared in situ by reaction of cyanothioacetamide with acetaldehyde in ethanolic piperidine) with compound 1 afforded the pyridinethione $16 \mathrm{~d}$. Compound $16 \mathrm{~d}$ was confirmed by spectroscopic data (IR, ${ }^{1} \mathrm{HNMR}$ ) and its chemical reactivity of this molecule to Gewald reaction with elemental sulfur. So, further reaction of compound $16 \mathrm{~d}$ with elemental sulfur in refluxing ethanol in the presence of little amount of triethylamine afforded the thieno[3,4-c]-pyridine derivative 18. Compound 18 was confirmed based on its elemental analysis and spectroscopic data. Thus, its IR spectrum showed disappearance of $(\mathrm{C} \equiv \mathrm{N})$ group at $v=2215 \mathrm{~cm}^{-1}$ of compound $16 \mathrm{~d}$ and appearance of $\mathrm{NH}_{2}$ group at $v=3440,3400 \mathrm{~cm}^{-1}$. Moreover, its ${ }^{1} \mathrm{HNMR}$ revealed the presence of a singlet signal at $\delta 6.57 \mathrm{ppm}$ assigned for thiophene proton, (Scheme 3). 


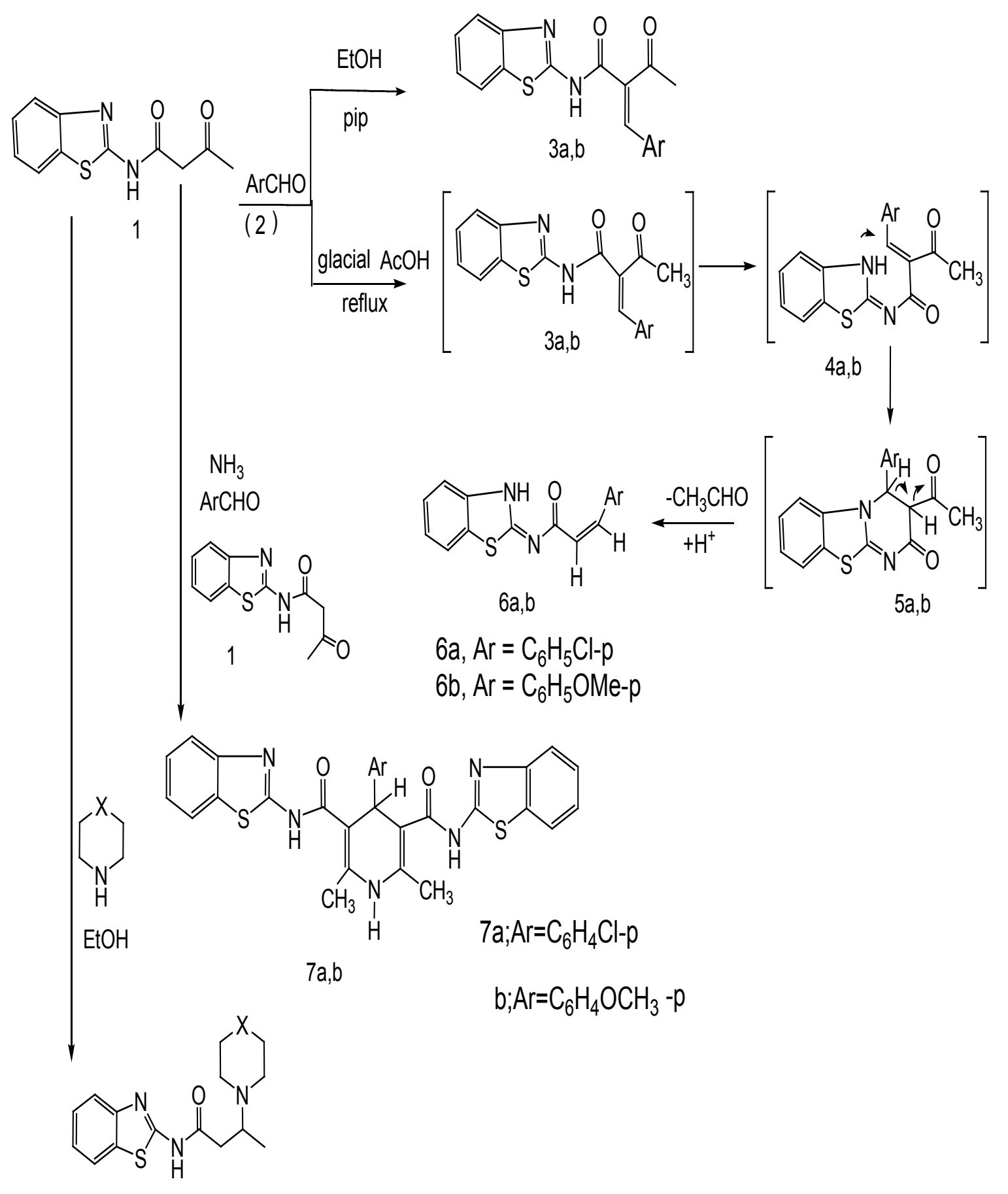

$8 a, b$

$$
\begin{aligned}
& 8 a, X=\mathrm{CH}_{2} \\
& 8 b, X=0
\end{aligned}
$$

Scheme 1. 
<smiles>CC1=C(C(=O)Nc2nc3ccccc3s2)Sc2ccccc2N1</smiles>

11<smiles>CCCCC(C)C(C)C</smiles>

$\mathrm{H}_{2} \mathrm{SO}_{4}$<smiles>Cc1cc(=O)nc2sc3ccccc3n12</smiles>

9

\section{$\mathrm{NH}_{2} \mathrm{OH} . \mathrm{HCl}$ $\mathrm{AcONa} / \mathrm{EtOH}$}<smiles>C/C(CC(=O)Nc1nc2ccccc2s1)=N\O</smiles>

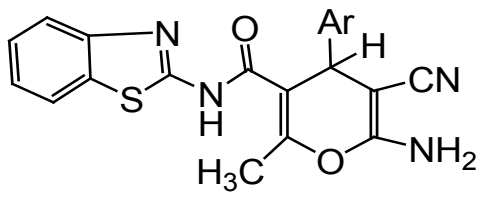

$13 a, b$

12,13a; $\mathrm{Ar}=\mathrm{C}_{6} \mathrm{H}_{4} \mathrm{OCH}_{3}(\mathrm{p})$ $b ; A r=\mathrm{C}_{6} \mathrm{H}_{4} \mathrm{Cl}(\mathrm{p})$

EtOH/pip/reflux<smiles>Cc1nc(O)c(C#N)c(Br)c1C(=O)Nc1nc2ccccc2s1</smiles>

14

Scheme 2.

The reactivity of pyridinethione 16 towards hydrazine hydrate as binucleophilic reagent was investigated. Thus, treatment of $16 \mathrm{a}$ with hydrazine hydrate in boiling ethanol afforded the pyrazolo[3,4-b]pyridine derivative 19. The IR spectrum of this reaction product showed the presence of absorption bands at $v=3426,3400$ $\mathrm{cm}^{-1}$ for $\mathrm{NH}_{2}$ and $3150 \mathrm{~cm}^{-1}$ for NH respectively, and disappearance of the cyano function group. Furthermore, compound 16a was reacted with ethylchloroacetate in refluxing ethanol containing sodium acetate to afford [5-(1,3-Benzothiazol- 2-ylcarbamoyl)-4-(4-chloro-phenyl)-3-cyano-6methyl-pyridin-2-ylsulfanyl]-acetic acid ethyl ester 20.

The structure of compound 20 was established based on the elemental analysis and spectral data. The IR spectrum of compound 20 exhibited the presence of the absorption band of cyano function group at $v=2201 \mathrm{~cm}^{-1}$. ${ }^{1} \mathrm{HNMR}$ spectrum of compound 20 revealed the presence of triplet at $\delta=1.16$ and quartet at $\delta=3.60 \mathrm{ppm}$ assigned to ethyl group in addition of $\mathrm{CH}_{2}$ group at $3.59 \mathrm{ppm}$ as singlet signal. 
Compound 20 was cyclized into the corresponding thieno[2,3-b]-pyridine derivative 21 upon boiling with ethanol solution containing a few drops of sodium ethoxide solution. The IR spectrum of compound 21 exhibited the absence of absorption band due to cyano function group and appearance of absorption bands due to $\mathrm{NH}_{2}$ function group at $v=3499,3450 \mathrm{~cm}^{-1}$. ${ }^{1} \mathrm{HNMR}$ spectrum of compound 21 revealed singlet signals at $\delta=0.83 \mathrm{ppm}$ assigned to $\mathrm{CH}_{3}$, triplet signals at $\delta=1.19$ ppm assigned to $\mathrm{CH}_{3}$, singlet signal at $2.42 \mathrm{ppm}$ assigned to $\mathrm{NH}_{2}$, quertet signals at $\delta=$ $3.67 \mathrm{ppm}$ assigned to $\mathrm{CH}_{2}$ in addition to aromatic protons and $\mathrm{NH}$ at $\delta 6.91-7.76 \mathrm{ppm}$. A solid evidence for the structure of compound 21 came from its synthesis by another route by conducting the reacting of $16 \mathrm{a}$ and ethyl chloroacetate in boiling solution of ethanolic sodium ethoxide (Scheme 3).

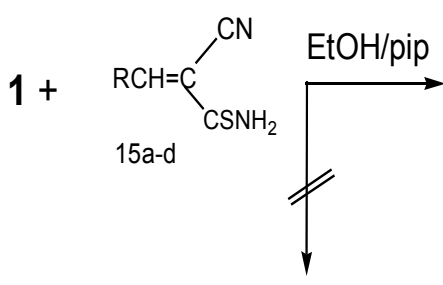<smiles>[R]C1C(C#N)=C(N)SC(C)=C1C(=O)Nc1nc2ccccc2s1</smiles>

17<smiles>[R]c1c(C(=O)Nc2nc3ccccc3s2)c(C)[nH]c(=S)c1C#N</smiles>

$15,16 \mathrm{a} ; \mathrm{R}=\mathrm{C}_{6} \mathrm{H}_{4} \mathrm{Cl}(\mathrm{p})$

b; $\mathrm{R}=\mathrm{C}_{6} \mathrm{H}_{4} \mathrm{Br}(\mathrm{p})$ $c ; \mathrm{R}=\mathrm{C}_{6} \mathrm{H}_{4} \mathrm{OCH}_{3}(\mathrm{p})$

$\mathrm{d} ; \mathrm{R}=\mathrm{CH}_{3}$

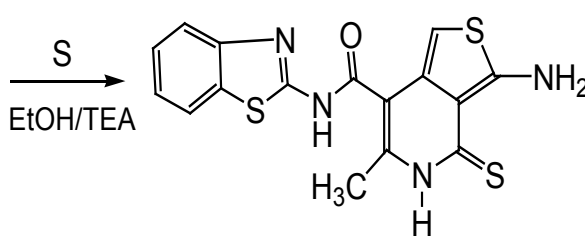

18<smiles></smiles>

Scheme 3. 
Furthermore, compound 1 readily reacted with cyanothioacetamide in refluxing ethanolic piperidine to yield the product that formulated as 6-(1,3-benzothiazol-2-ylamino)-4-methyl-2-thioxo1,2-dihydro-pyridine-3-carbonitrile 22. Compound 22 was confirmed based on spectral data. Its IR spectrum revealed the absence of carbonyl group characteristic to the acetoacetanilide and presence of $(\mathrm{C} \equiv \mathrm{N})$ group at $\vee 2203 \mathrm{~cm}^{-1}$, (Scheme 4). Fusing compound 1 with malononitrile over melting point without solvent in the presence of ammonium acetate or refluxing in ethanolic piperidine afforded the pyridone (23a) in quantitative yield. Compound 23a was established based on its spectral data. Also, the pyridone derivative $23 \mathrm{~b}$ was obtained by reacting of compound 1 with cyanoacetamide under the same reaction conditions, (Scheme 4).<smiles>Cc1cc(Nc2nc3ccccc3s2)[nH]c(=S)c1C#N</smiles>

22<smiles>CCOCCCCCC(N)=S</smiles>

EtOH/pip
1<smiles>[X]C(N)=O</smiles>

or EtOH/pip<smiles>[X]c1c(C)cc(=O)n(-c2nc3ccccc3s2)c1N</smiles>

23a,b $\mathrm{x}=\mathrm{CN}$<smiles>CC(=O)CC(C)CC(C)C(=O)Nc1nc2ccccc2s1</smiles><smiles>Cc1c(CC(=O)O)c(=O)nc2sc3ccccc3n12</smiles>

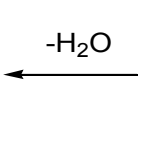<smiles>CCOC(=O)C/C(C(=Nc1nc2ccccc2s1)C(=O)O)=C(/C)O</smiles>

27

24,28a; $\mathrm{Ar}=\mathrm{C}_{6} \mathrm{H}_{4} \mathrm{Br}-\mathrm{p}$ $\mathrm{b} ; \mathrm{Ar}=\mathrm{C}_{6} \mathrm{H}_{4} \mathrm{CH}_{3-\mathrm{p}}$

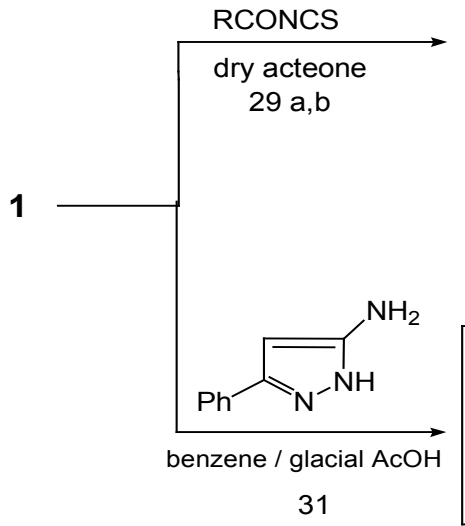

31
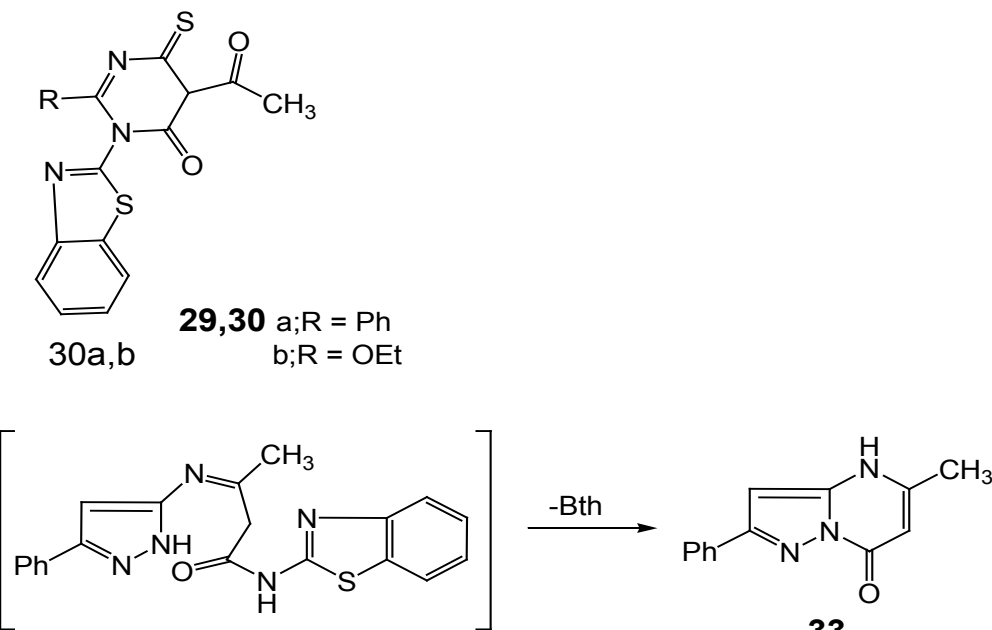

32 
The reaction of compound 1 with $\omega$-bromoacetophenones 24 afforded pyrimido[2,1-b][1,3]-benzothiazol-2-one derivatives $28 \mathrm{a}, \mathrm{b}$. Compound 28 was established by analytical data (IR, ${ }^{1} \mathrm{HNMR}$ ) and elemental analysis. Compound 28 was assumed to be proceeding via the first step involves a carbon carbon bond forming reaction by the attack of carbanion generated by treatment of acetoacetanilide 1 with sodium hydride in dry benzene on the bromomethyl to obtain the corresponding sodium salt. This in turn when treated with $\omega$-bromoacetophenones 24 afforded the intermediate 26 which cyclized in the reaction medium by loss of $\mathrm{H}_{2} \mathrm{O}$ to the final product 28 , (Scheme 4).

Treatment of 1 with benzoyl and ethoxycarbonyl isothiocyanate 29a,b (prepared in situ) in dry acetone afforded the pyrimidine derivatives $30 \mathrm{a}, \mathrm{b}$. Compound 30 was confirmed by spectral data, IR, ${ }^{1} \mathrm{HNMR}$ and elemental analysis, (Scheme 12). The reaction of compound 1 with aminopyrazole 33 in benzene containing glacial acetic acid afforded products via water and 2-aminobenzo-thiazole molecule elimination. The pyrazolopyrimidine 33 was established as reaction product based on spectral data. Thus, the mass spectrum revealed a molecular ion peak at $\mathrm{m} / \mathrm{z}=225\left(\mathrm{M}^{+}\right)$corresponding to the molecular formula $\mathrm{C}_{13} \mathrm{H}_{11} \mathrm{~N}_{3} \mathrm{O}$. Also, the ${ }^{1} \mathrm{HNMR}$ spectrum recorded in (DMSO-d $)_{6}$ exhibited a singlet signal at $\delta=2.31 \mathrm{ppm}$ assigned to $\mathrm{CH}_{3}$, singlet signal at $\delta=5.61 \mathrm{ppm}$ assigned to pyrazole- $\mathrm{H}$, singlet signal at $\delta=6.58 \mathrm{ppm}$ assigned to pyrimidine- $\mathrm{H}$, multiplet signals at $\delta=7.40-8.00 \mathrm{ppm}$ assigned to $\mathrm{Ar}-\mathrm{H}$, and hump signal at $\delta=12.57 \mathrm{ppm}$ assigned to $\mathrm{NH}$. Compound 33 is assumed to be formed via initial condensation of exocyclic amino function in 31 with the carbonyl group in compound 1 to give the intermediate 32 which readily cyclized to the final isolable product pyrazolo[1,5-a]pyrimidine 33, (Scheme 4).

Coupling of compound 1 with diazonium salt of compounds $34 \mathrm{a}, \mathrm{b}$ yielding the coupling products. 7-Amino-4-methyl-8-[4-(thiazol-2-ylsulfamoyl)-phenylazo]-pyrazolo[5,1-c][1,2,4] triazine-(1,3-benzothiazol-2-yl)-3-carboxamide 37a and 4-Methyl-7-oxo-8-[4-(guanidin-2-ylsulfamoyl)-phenylazo]-pyrazolo[5,1-c]-[1,2,4] triazine-(1,3-benzothiazol-2-yl)-3-carboxamide $37 \mathrm{~b}$, in good yield via the intermediates $36 \mathrm{a}, \mathrm{b}$ respectively, (Scheme 5).

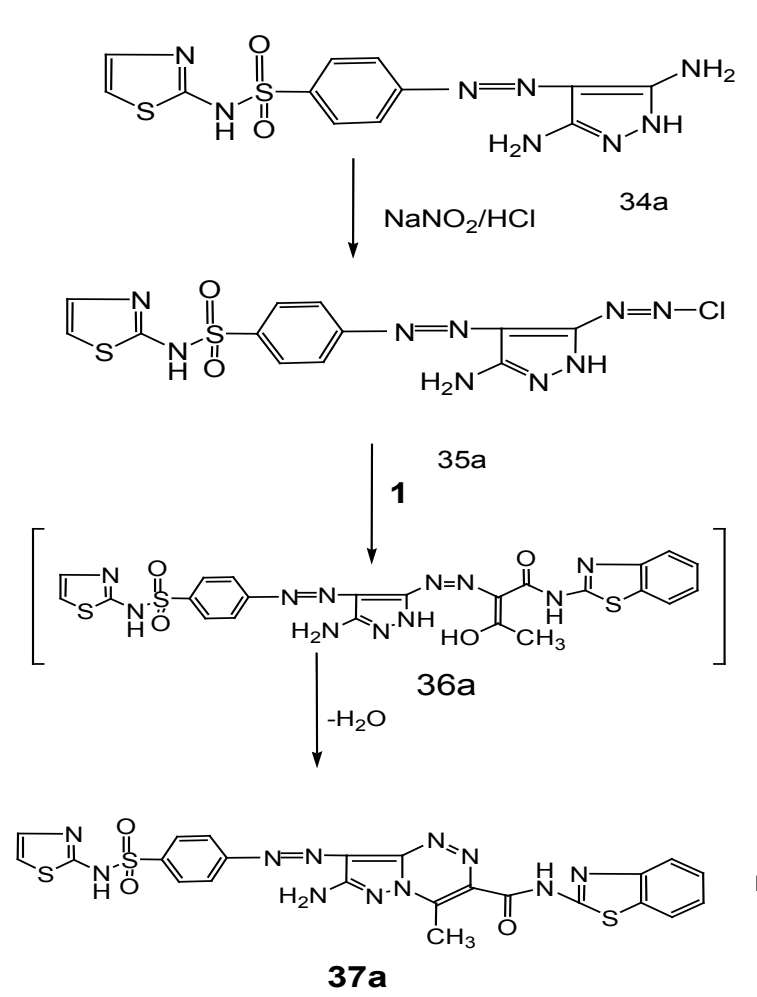

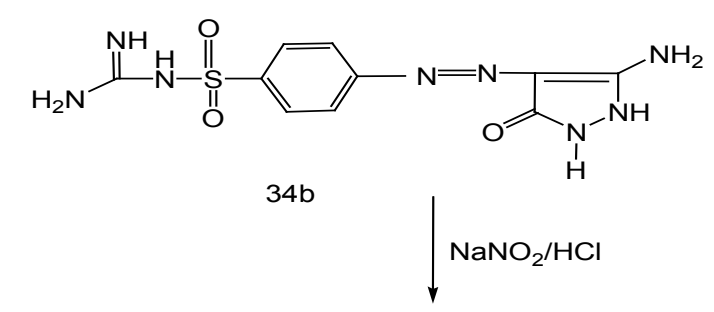<smiles>N=C(N)NS(=O)(=O)c1ccc(N=Nc2c(N=NCl)[nH][nH]c2=O)cc1</smiles>
1

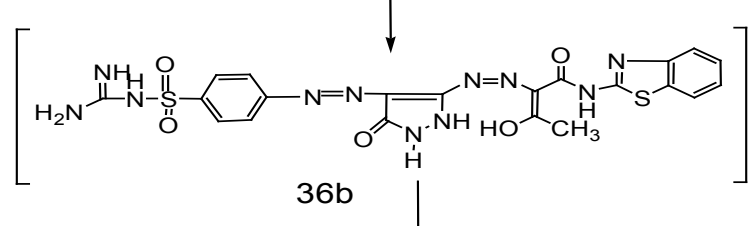
$36 b$<smiles></smiles>

37b

Scheme 5 . 


\section{Materials and Methods}

All melting points are uncorrected. IR spectra $(\mathrm{KBr})$ were recorded on a FTIR 5300 spectrometer $\left(v, \mathrm{~cm}^{-1}\right)$. The ${ }^{1} \mathrm{H}$ NMR spectra were recorded in DMSO- $\mathrm{d}_{6}$ and $\mathrm{CDCl}_{3}$ at $200,400 \mathrm{MHz}$ on a Varian Gemini NMR spectrometer $(\delta, \mathrm{ppm})$ using TMS as an internal standard. Mass spectra were obtained on GC Ms-QP 1000 EX mass spectrometer at 70 ev. Elemental analysis was carried out by the Microanalytical Research Center, Faculty of Science, Cairo University and Microanalytical Research Center, Assiut University.

Preparation of compounds (6a,b): General procedure

To a solution of acetoacetanilide 1 (0.01 mole) in glacial acetic acid $(30 \mathrm{ml})$, aromatic aldehyde $(0.01$ mole $)$ was added. The reaction mixture was refluxed for $24 \mathrm{hrs}$ and left to cool. The solid product was collected by filteration and recrystallized from the proper solvent to give $6 \mathrm{a}, \mathrm{b}$.

N-1,3-Benzothiazol-2-yl-3-(4-chlorophenyl) acrylamide (6a)

It was obtained as a pale yellow crystals from ethanol; yield $83 \%$; m.p. $280-2^{\circ} \mathrm{C}$; IR (KBr) $v \mathrm{~cm}^{-1}$ $3150(\mathrm{NH}), 3050(\mathrm{CH}-$ arom $), 1686(\mathrm{C}=\mathrm{O}) ;{ }^{1} \mathrm{H}$ NMR (DMSO-d $\left.{ }_{6}\right) \delta=6.9-7.0$ (d, 1H, CH-olefinic), 7.3-7.9 (m, 8H, Ar-H), 8.0-8.1 (d, 1H, CH-olefinic), 12.63 (s, 1H, NH); MS: m/z 312 (M-2). Found; C, 61.07; H, 3.33; N, 8.92; cald. For $\mathrm{C}_{16} \mathrm{H}_{11} \mathrm{ClN}_{2} \mathrm{OS}$ (314.79); C, 61.05; H, 3.52; N, 8.90.

\section{N-1,3-Benzothiazol-2-yl-3-(4-methoxyphenyl)} acrylamide (6b)

It was obtained as colorless crystals from ethanol; yield $77 \%$; m.p. $256-8^{\circ} \mathrm{C}$; IR (KBr) v $\mathrm{cm}^{-1} 3174(\mathrm{NH}), 3062(\mathrm{CH}$-arom), $2957(\mathrm{CH}-$ aliph), $1680(\mathrm{C}=\mathrm{O}) ;{ }^{1} \mathrm{H}$ NMR (DMSO-d ${ }_{6}$ ) $\delta=3.82$ (s, 3H, $\mathrm{OCH}_{3}$ ), 6.79-6.84 (d, 1H, CH-olefinic) $\mathrm{J}=$ $10.4 \mathrm{~Hz}, 7.02-7.77$ (m, 8H, Ar-H), 7.97-8.00 (d, $1 \mathrm{H}, \mathrm{CH}$-olefinic), 12.5 (s, 1H, NH). Found; C, 65.77; H, 4.54; N, 9.02; cald. For; $\mathrm{C}_{17} \mathrm{H}_{14} \mathrm{~N}_{2} \mathrm{O}_{2} \mathrm{~S}$ (310.37); C, 65.79; H, 4.55; N, 9.03.

Preparation of compounds (7a,b): General procedure

A mixture of acetoacetanilide 1 (0.02 mole), $20 \%$ aqueous $\mathrm{NH}_{3}(1.2 \mathrm{ml})$ and aromatic aldehydes $(0.01 \mathrm{~mole})$ in $(20 \mathrm{ml}) \mathrm{EtOH}$ was refluxed for 12 $\mathrm{hr}$. The solid product which produced on hot was collected by filteration and recrystallized from the proper solvent to give $7 \mathrm{a}, \mathrm{b}$.
4-(4-Chloro-phenyl)-2,6-dimethyl-1,4-dihydropyridine-3,5-dicarboxamide bis(1,3-benzothiazol-2yl) (7a)

It was obtained as yellow crystals from Dioxane/EtOH; yield $83 \%$; m.p. $290-2^{\circ} \mathrm{C}$; IR $(\mathrm{KBr}) \vee \mathrm{cm}^{-1} 3330,3230(2 \mathrm{NH}), 3060$ (CH-arom), 2980 (CH-aliphatic), $1657 \quad(\mathrm{C}=\mathrm{O}) ;{ }^{1} \mathrm{H}$ NMR $\left(\mathrm{DMSO}_{6}\right) \delta=2.26\left(\mathrm{~s}, 6 \mathrm{H}, 2 \mathrm{CH}_{3}\right), 5.37(\mathrm{~s}, 1 \mathrm{H}$, pyridine-H), 7.14-7.93 (m, 12H, Ar-H), 9.05 (s, $1 \mathrm{H}, \mathrm{NH}), 11.93$ (s, 2H, 2NH). Found; C, 60.89; $\mathrm{H}, 3.89$; N, 12.25; cald. For $\mathrm{C}_{29} \mathrm{H}_{22} \mathrm{ClN}_{5} \mathrm{O}_{2} \mathrm{~S}_{2}$ (572.11); C, 60.88; H, 3.88; N, 12.24 .

4-(4-Methoxy-phenyl)-2,6-dimethyl-1,4dihydropyridine-3,5-dicarboxamide bis(1,3benzothiazol-2-yl) (7b)

It was obtained as pale yellow crystals from benzene; yield $67 \%$; m.p. $158-60^{\circ} \mathrm{C}$; IR $(\mathrm{KBr})$ $v \mathrm{~cm}^{-1}$ 3320, $3250(2 \mathrm{NH}), 2956$ (CH-aliphatic), $1668(\mathrm{C}=\mathrm{O})$. Found; $\mathrm{C}, 63.48 ; \mathrm{H}, 4.45 ; \mathrm{N}, 12.36$; cald. For $\mathrm{C}_{30} \mathrm{H}_{25} \mathrm{~N}_{5} \mathrm{O}_{3} \mathrm{~S}_{2}$ (567.69); C, 63.47; $\mathrm{H}$, 4.44; N, 12.34 .

Preparation of compounds (8a,b): General procedure

A solution of acetoacetanilide $\mathbf{1}$ in ethanol (30 $\mathrm{ml}$ ) was treated with a few drops of piperidine $(0.5 \mathrm{ml})$, or morpholin $(0.5 \mathrm{ml})$. The solution was heated under reflux for $1 \mathrm{hr}$. The solid product which produced on hot was collected by filteration and recrystallized from the proper solvent to give $8 \mathrm{a}, \mathrm{b}$.

N-1,3-Benzothiazol-2-yl-3-piperidin-1-ylbut-2enamide (8a)

It was obtained as yellow crystals from ethanol; yield $76 \%$; m.p. $232-4^{\circ} \mathrm{C}$; IR ( $\left.\mathrm{KBr}\right) \mathrm{v}$ $\mathrm{cm}^{-1} 3200(\mathrm{NH}), 3025$ (CH-arom), $2937(\mathrm{CH}-$ aliph), $1659(\mathrm{C}=\mathrm{O}) ;{ }^{1} \mathrm{H}$ NMR (DMSO-d $\left.{ }_{6}\right) \delta=$ $1.52\left(\mathrm{~s}, 3 \mathrm{H}, \mathrm{CH}_{3}\right), 2.96-3.54\left(\mathrm{~m}, 10 \mathrm{H}, 5 \mathrm{CH}_{2}\right)$, $5.17(\mathrm{~s}, 1 \mathrm{H}, \mathrm{CH}=\mathrm{C}), 7.18-7.88(\mathrm{~m}, 5 \mathrm{H}, \mathrm{Ar}-\mathrm{H}$ and $\mathrm{NH}) ; \mathrm{MS}: \mathrm{m} / \mathrm{z}=301\left(\mathrm{M}^{+}\right)$. Found; $\mathrm{C}, 63.77 ; \mathrm{H}$, 6.36; N, 13.95; cald. For $\mathrm{C}_{16} \mathrm{H}_{19} \mathrm{~N}_{3} \mathrm{OS}(301.41) ; \mathrm{C}$, $63.67 ; \mathrm{H}, 6.35 ; \mathrm{N}, 13.94$.

N-1,3-Benzothiazol-2-yl-3-morpholin-4-yl-but-2enamide (8b)

It was obtained as colorless crystals from ethanol; yield $81 \%$; m.p. $222^{\circ} \mathrm{C}$; IR $(\mathrm{KBr}) v \mathrm{~cm}^{-1}$ $3200(\mathrm{NH}), 2918$ (CH-aliph), $1655(\mathrm{C}=\mathrm{O}) ;{ }^{1} \mathrm{H}$ NMR $\left(\right.$ DMSO-d $\left._{6}\right) \delta=2.48\left(\mathrm{~s}, 3 \mathrm{H}, \mathrm{CH}_{3}\right), 3.27-$ $3.67\left(\mathrm{~m}, 8 \mathrm{H}, 4 \mathrm{CH}_{2}\right), 5.19(\mathrm{~s}, 1 \mathrm{H}, \mathrm{CH}=\mathrm{C}), 7.21-$ 7.90 (m, 4H, Ar-H), 11.52 (s, 1H, NH). Found; C, 59.39; H, 5.65; N, 13.86; cald. For $\mathrm{C}_{15} \mathrm{H}_{17} \mathrm{~N}_{3} \mathrm{O}_{2} \mathrm{~S}$ (303.39); C, 59.39; H, 5.67; N, 13.85. 
4-Methyl-2H-pyrimido[2,1-b][1,3]-benzothiazol2-one (9)

Acetoacetanilide 1 (2 gm) was heated under reflux in conc. sulfuric acid for 10 minutes at $250^{\circ} \mathrm{C}$. The reaction mixture was left to stand, and poured into $\mathrm{H}_{2} \mathrm{O}(100 \mathrm{ml})$ the solid product was collected by filteration and recrystallized from $\mathrm{DMF} / \mathrm{EtOH}$ to give $(\mathbf{9} ; 62 \%)$ as brown crystals; m.p. $>300^{\circ} \mathrm{C}$; IR (KBr) $v \mathrm{~cm}^{-1} 3042$ (CH-arom), 2969 (CH-aliph), 1649 (C=O). Found; C, 61.08; $\mathrm{H}, 3.72$; N, 12.94; cald. For $\mathrm{C}_{11} \mathrm{H}_{8} \mathrm{~N}_{2} \mathrm{OS}$ (216.26); C, 61.09; H, 3.73; N, 12.95 .

N-1,3-Benzothiazol-2-yl-3-(hydroxyimino) butanamide (10)

To a solution of acetoacetanilide 1 (0.01 mole), hydroxylamine hydrochloride ( 0.01 mole), sodium acetate $(0.5 \mathrm{gm}), \mathrm{H}_{2} \mathrm{O}(1 \mathrm{ml})$ and $20 \mathrm{ml}$ of ethanol was refluxed for $3 \mathrm{hrs}$. Then left to cool, and poured into crushed ice. The solid product was collected by filteration and recrystallized from Dioxane/EtOH to give (10, 72\%) as brown crystals; m.p. $>300^{\circ} \mathrm{C}$; IR (KBr) $v$ cm$^{-1} 3440(\mathrm{OH})$, 3348 (NH), 2921 (CH-aliph), $1650(\mathrm{C}=\mathrm{O}) ;{ }^{1} \mathrm{H}$ NMR (DMSO-d $) \delta=1.23\left(\mathrm{~s}, 3 \mathrm{H}, \mathrm{CH}_{3}\right), 3.67$ (s, 2H, $\mathrm{CH}_{2}$ ), 7.30-7.41 (m, 5H, 4Ar-H + NH), 8.12 (hump, $1 \mathrm{H}, \mathrm{OH}$ ). Found $\mathrm{C}_{11} \mathrm{H}_{11} \mathrm{~N}_{3} \mathrm{O}_{2} \mathrm{~S}$ (249.29); $\mathrm{C}$, 53.02; H, 4.46; N, 16.88; cald. For; C, 53.00; H, $4.45 ; \mathrm{N}, 16.86$.

N-1,3-Benzothiazol-2-yl-3-methyl-4H-1,4benzothiazine-2-carboxamide (11)

To a solution of 1 ( 0.01 mole) and $2.5 \mathrm{ml}$ (0.032 mole) of triethylamine in ethanol (30 $\mathrm{ml})$, o-aminothiophenol (0.01 mole) in $7.5 \mathrm{ml}$ of ethanol was added at $20^{\circ} \mathrm{C}$. The solution was kept at room temperature for $6 \mathrm{~h}$, then poured into water. The solid product was collected by filteration and recrystallized from ethanol to give $(11,81 \%)$ as orange crystals; m.p. $202^{\circ} \mathrm{C}$; IR $(\mathrm{KBr}) \vee \mathrm{cm}^{-1} 3255(\mathrm{NH}), 3050$ (CH-arom), $1660(\mathrm{C}=\mathrm{O}) ;{ }^{1} \mathrm{H}$ NMR (DMSO-d $) ~ \delta=2.28(\mathrm{~s}$, $\left.3 \mathrm{H}, \mathrm{CH}_{3}\right), 6.74-7.93$ (m, 9H, Ar-H and NH), 8.94 (hump, 1H, NH). Found; C, 60.17; H, 3.87; N, 12.39; cald. For $\mathrm{C}_{17} \mathrm{H}_{13} \mathrm{~N}_{3} \mathrm{OS}_{2}$ (339.44); C, 60.15; H, 3.86; N, 12.38 .

\section{Preparation of compounds (13a,b):General procedure}

A mixture of 1 (0.01 mole), $\alpha$-cyano-4substituted cinnamonitrile $(0.01$ mole $)$ in ethanol (30 $\mathrm{ml})$ was treated with a few drops of piperidine and heated under reflux for $8 \mathrm{hrs}$. The reaction mixture allowed to cool, poured into crushed ice and acidified with $\mathrm{HCl}$. The solid product was filtered off and recrystallized from the proper solvent to give $13 \mathrm{a}, \mathrm{b}$.

6-Amino-N-1,3-benzothiazol-2-yl-5-cyano4-(4-methoxy-phenyl)-2-methyl-4H-pyran-3carboxamide (13a)

It was obtained as yellow crystals from ethanol; yield $81 \%$; m.p. $264-66^{\circ} \mathrm{C}$; IR ( $\left.\mathrm{KBr}\right) v$ $\mathrm{cm}^{-1}$ 3430, $3341\left(\mathrm{NH}_{2}\right), 3086$ (CH-arom), 2917 (CH-aliph), $2202(\mathrm{C} \equiv \mathrm{N}), 1682(\mathrm{C}=\mathrm{O}) ;{ }^{1} \mathrm{H}$ NMR $\left(\mathrm{DMSO}_{6} \mathrm{~d}_{6}\right) \delta=2.19\left(\mathrm{~s}, 3 \mathrm{H}, \mathrm{CH}_{3}\right), 3.79(\mathrm{~s}, 3 \mathrm{H}$, $\left.\mathrm{OCH}_{3}\right), 5.71$ (s, 1H, 4H-pyrane), 7.52-8.18 (m, $11 \mathrm{H}, \mathrm{Ar}-\mathrm{H}, \mathrm{NH}_{2}$ and $\mathrm{NH}$ ). Found; C, 60.15; H, 4.35; N, 13.40; cald. For $\mathrm{C}_{22} \mathrm{H}_{18} \mathrm{~N}_{4} \mathrm{O}_{3} \mathrm{~S}$ (418.48); C, 63.14; H, 4.34; N, 13.39.

6-Amino-N-1,3-benzothiazol-2-yl-4-(4-chlorophenyl)-5-cyano-2-methyl-4H-pyran-3carboxamide (13b)

It was obtained as brown crystals from ethanol; yield $86 \%$; m.p. $184^{\circ} \mathrm{C}$; IR $(\mathrm{KBr}) \vee \mathrm{cm}^{-1}$ 3420, 3331, $3108\left(\mathrm{NH}_{2}, \mathrm{NH}\right), 2924$ (CH-aliph), $2200(\mathrm{C} \equiv \mathrm{N}), 1677(\mathrm{C}=\mathrm{O}) ;{ }^{1} \mathrm{H}$ NMR $($ DMSO-d $)$ $\delta=2.20\left(\mathrm{~s}, 3 \mathrm{H}, \mathrm{CH}_{3}\right), 5.72$ (s, 1H, 4H-pyrane), 7.29-8.18 (m, 11H, Ar-H, $\mathrm{NH}_{2}$ and $\left.\mathrm{NH}\right)$. Found; C, 59.65; H, 3.59; N, 13.26; cald. For $\mathrm{C}_{21} \mathrm{H}_{15} \mathrm{ClN}_{4} \mathrm{O}_{2} \mathrm{~S}$ (422.90); C, 59.64; H, 3.58; N, 13.25.

Preparation of compounds (16a-d): General procedure

A mixture of $1 \quad(0.01$ mole $)$, arylidinecyanothioacetamide $(0.01$ mole $)$ in ethanol $(30 \mathrm{ml})$ was treated with a few drops of piperidine and refluxed for $5 \mathrm{hrs}$. The reaction mixture then left to cool, poured into crushed ice and acidified with $\mathrm{HCl}$. The solid product was collected and recrystallized from the proper solvent to give $16 \mathrm{a}-\mathrm{d}$.

N-1,3-Benzothiazol-2-yl-4-(4-chlorophenyl)-5cyano-2-methyl-6-thioxo-1,6-dihydropyridine-3carboxamide (16a)

It was obtained as yellow crystals from ethanol; yield $86 \%$; m.p. $210^{\circ} \mathrm{C}$; IR $(\mathrm{KBr}) \vee \mathrm{cm}^{-1}$ 3217 (NH), 3058 (CH-arom), 2973 (CH-aliph), $2200(\mathrm{CN}), 1673(\mathrm{C}=\mathrm{O}) ;{ }^{1} \mathrm{H}$ NMR $\left(\mathrm{DMSO}_{\mathrm{d}}\right) \delta=$ 1.92 (s, 3H, $\left.\mathrm{CH}_{3}\right), 7.27-7.95$ (m, 8H, Ar-H), 12.77 (s, 1H, NH), 14.56 (s, 1H, NH). Found; C, 57.74; $\mathrm{H}, 3.01 ; \mathrm{N}, 12.83$; cald. For $\mathrm{C}_{21} \mathrm{H}_{13} \mathrm{ClN}_{4} \mathrm{OS}_{2}$ (436.94); C, 57.73; H, 3.00; N, 12.82.

N-1,3-Benzothiazol-2-yl-4-(4-bromophenyl)-5cyano-2-methyl-6-thioxo-1,6-dihydropyridine-3carboxamide (16b)

It was obtained as pale yellow crystals from benzene; yield $81 \%$; m.p. $198-200^{\circ} \mathrm{C}$; IR (KBr) 
$v \mathrm{~cm}^{-1}$ 3380, $3215(2 \mathrm{NH}), 3056(\mathrm{CH}-$ arom $), 2947$ (CH-aliph), $2172(\mathrm{C} \equiv \mathrm{N}), 1654(\mathrm{C}=\mathrm{O})$. Found; $\mathrm{C}$, 52.42; H, 2.73; N, 11.65; cald. For $\mathrm{C}_{21} \mathrm{H}_{13} \mathrm{BrN}_{4} \mathrm{OS}_{2}$ (481.40); C, 52.40; H, 2.72; N, 11.64 .

N-1,3-Benzothiazol-2-yl-5-cyano-4-(4methoxyphenyl)-2-methyl-6-thioxo-1,6dihydropyridine-3-carboxamide (16c)

It was obtained as yellow crystals from benzene; yield $85 \%$; m.p. $250^{\circ} \mathrm{C}$; IR $(\mathrm{KBr}) \mathrm{v}$ $\mathrm{cm}^{-1}$ 3447, 3182 (2NH), 3065 (CH-arom), 2959 (CH-aliph), $2230(\mathrm{C} \equiv \mathrm{N}), 1686(\mathrm{C}=\mathrm{O}) .{ }^{1} \mathrm{H} \mathrm{NMR}$ $\left(\mathrm{DMSO}_{6}\right) \delta=2.43\left(\mathrm{~s}, 3 \mathrm{H}, \mathrm{CH}_{3}\right), 3.70(\mathrm{~s}, 3 \mathrm{H}$, $\left.\mathrm{OCH}_{3}\right), 6.97-7.96(\mathrm{~m}, 10 \mathrm{H}, \mathrm{Ar}-\mathrm{H}+2 \mathrm{NH})$. Found; C, 61.08; H, 3.72; N, 12.94; cald. For $\mathrm{C}_{22} \mathrm{H}_{16} \mathrm{~N}_{4} \mathrm{O}_{2} \mathrm{~S}_{2}$ (432.53); C, 61.09; H, 3.73; N, 12.95 .

N-1,3-Benzothiazol-2-yl-5-cyano-2,4-dimethyl-6thioxo-1,6-dihydropyridine-3-carboxamide (16d)

It was obtained as brown crystals from ethanol; yield 57\%; m.p. $228-230^{\circ} \mathrm{C}$; IR (KBr) $v \mathrm{~cm}^{-1} 3422$ (NH), 3062 (CH-arom), 2929 (CH-aliph), 2215 $(\mathrm{CN}), 1680(\mathrm{C}=\mathrm{O}) .{ }^{1} \mathrm{H}$ NMR $\left(\mathrm{CDCl}_{3}\right) \delta=0.73(\mathrm{~s}$, $\left.3 \mathrm{H}, \mathrm{CH}_{3}\right), 1.65\left(\mathrm{~s}, 3 \mathrm{H}, \mathrm{CH}_{3}\right), 6.74-7.29(\mathrm{~m}, 6 \mathrm{H}$, Ar-H and 2NH). Found; C, 56.46; H, 3.57; N, 16.47; cald. For $\mathrm{C}_{16} \mathrm{H}_{12} \mathrm{~N}_{4} \mathrm{OS}_{2}$ (340.43); C, 56.45; $\mathrm{H}, 3.55$; N, 16.46 .

3-Amino-N-1,3-benzothiazol-2-yl-6-methyl4-thioxo-4,5-dihydrothieno[3,4-c]pyridine-7carboxamide (18)

Equimolar amounts of $16 \mathrm{~d}$ (0.01 mole) and elemental sulfur $(0.01$ mole $)$ in ethanol $(30 \mathrm{ml})$ were treated with triethylamine $(0.5 \mathrm{ml})$ and refluxed for $4 \mathrm{hrs}$. The solid formed after cooling was collected and recrystallized from benzene to give $(18 ; 60 \%)$ as green crystals; m.p. $180^{\circ} \mathrm{C}$; IR $(\mathrm{KBr}) \vee \mathrm{cm}^{-1} 3440,3400\left(\mathrm{NH}_{2}\right), 3180,3120$ (2NH), 3050 (CH-arom), 2937 (CH-aliph), $1690(\mathrm{C}=\mathrm{O}) ;{ }^{1} \mathrm{H}$ NMR (DMSO-d $) \delta=1.71$ $\left(\mathrm{s}, 3 \mathrm{H}, \mathrm{CH}_{3}\right), 6.45\left(\mathrm{~s}, 2 \mathrm{H}, \mathrm{NH}_{2}\right), 6.57(\mathrm{~s}, 1 \mathrm{H}$, thiophene-H), 6.86-6.87 (m, 5H, Ar-H and $\mathrm{NH})$, 9.84 (s, 1H, NH). Found; C, 51.60; H, 3.27; N, 15.05; cald. For $\mathrm{C}_{16} \mathrm{H}_{12} \mathrm{~N}_{4} \mathrm{OS}_{3}$ (372.49); C, 51.59; H, 3.25; N, 15.04 .

3-Amino-N-1,3-benzothiazol-2-yl-4-(4chlorophenyl)-6-methyl-1H-pyrazolo [3,4-b] pyridine-5-carboxamide (19)

A solution of compound $16 \mathrm{a}$ ( 0.01 mole) in ethanol $(10 \mathrm{ml})$ was treated with an excess of hydrazine hydrate $(10 \mathrm{ml})$. The mixture was heated under reflux for $12 \mathrm{hrs}$, and left to stand. Poured on ice cold water and acidified with $\mathrm{HCl}$. The solid product so formed was obtained by filteration and Egypt.J.Chem. 61, No.6 (2018) recrystallized from ethanol to give $(19 ; 82 \%)$ as brown crystals; m.p. $154-6^{\circ} \mathrm{C}$; IR $(\mathrm{KBr}) \vee \mathrm{cm}^{-1}$ 3426, $3400\left(\mathrm{NH}_{2}\right), 3150(\mathrm{NH}), 3050$ (CH-arom), 2927 (CH-aliph), $1677(\mathrm{C}=\mathrm{O}) ;{ }^{1} \mathrm{H}$ NMR $\left(\mathrm{CDCl}_{3}\right)$ $\delta=1.16\left(\mathrm{~s}, 3 \mathrm{H}, \mathrm{CH}_{3}\right), 3.68\left(\mathrm{~s}, 2 \mathrm{H}, \mathrm{NH}_{2}\right), 6.67-7.77$ (m, 10H, Ar-H and 2NH). Found; C, 58.01; H, 3.49 ; N, 19.33; cald. For $\mathrm{C}_{21} \mathrm{H}_{15} \mathrm{ClN}_{6} \mathrm{OS}$ (434.91); C, 58.00; H, 3.48; N, 19.32 .

[5-(1,3-Benzothiazol-2-yl-carbamoyl)-4-(4chlorophenyl)-3-cyano-6-methyl-pyridin-2-ylsulfanyl]-acetic acid ethyl ester (20)

To a solution of $16 \mathrm{a}$ ( $0.01 \mathrm{~mole})$ in ethanol, ethylchloroacetate $(0.01$ mole $)$ and sodium acetate $(0.5 \mathrm{~g})$ were added. The mixture was refluxed for 4 hrs, cooled, poured into crushed ice and acidified with $\mathrm{HCl}$. The precipitate formed was collected and recrystallized from ethanol to give (20; 73\%) as pale yellow crystals; m.p. $158-60^{\circ} \mathrm{C}$; IR $(\mathrm{KBr})$ $v \mathrm{~cm}^{-1} 3258(\mathrm{NH}), 3061$ (CH-arom), $2924(\mathrm{CH}-$ aliph), $2201(\mathrm{C} \equiv \mathrm{N}), 1734,1667(2 \mathrm{C}=\mathrm{O}) ;{ }^{1} \mathrm{H}$ NMR $\left(\mathrm{CDCl}_{3}\right) \delta=1.10\left(\mathrm{~s}, 3 \mathrm{H}, \mathrm{CH}_{3}\right), 1.16\left(\mathrm{t}, 3 \mathrm{H}, \mathrm{CH}_{3}\right)$, 3.59 (s, 2H, $\left.\mathrm{CH}_{2}\right), 3.60$ (q, 2H, $\left.\mathrm{CH}_{2}\right), 7.10-7.70$ (m, 8H, Ar-H), 8.90 (s, 1H, NH). Found; C, 57.43; $\mathrm{H}, 3.67$; N, 10.72; cald. For $\mathrm{C}_{25} \mathrm{H}_{19} \mathrm{ClN}_{4} \mathrm{O}_{3} \mathrm{~S}_{2}$ (523.04); C, 57.41; H, 3.66; N, 10.71.

3-Amino-5-(1,3-benzothiazol-2-yl-carbamoyl)4-(4-chloro-phenyl)-6-methyl-thieno [2,3-b] pyridine-2-carboxylic acid ethyl ester (21):

$\operatorname{Method}(A)$

To a solution of sodium ethoxide, a solution of 20 ( 0.01 mole $)$ in ethanol $(20 \mathrm{ml})$ was added. The reaction mixture was refluxed for $3 \mathrm{hrs}$. After cooling the reaction mixture was poured into cold water $(50 \mathrm{ml})$ and acidified with $\mathrm{HCl}$. The product formed was collected by filteration and recrystallized from ethanol to give $(21 ; 65 \%)$ as yellow crystals; m.p. $186-88^{\circ} \mathrm{C}$; IR $(\mathrm{KBr}) \vee \mathrm{cm}^{-1}$ 3499, $3450\left(\mathrm{NH}_{2}\right), 3340(\mathrm{NH}), 2929$ (CH-aliph), $1683(\mathrm{C}=\mathrm{O}) ;{ }^{1} \mathrm{H}$ NMR $\left(\mathrm{CDCl}_{3}\right) \delta=0.83(\mathrm{~s}, 3 \mathrm{H}$, $\left.\mathrm{CH}_{3}\right), 1.19\left(\mathrm{t}, 3 \mathrm{H}, \mathrm{CH}_{3}\right), 2.42\left(\mathrm{~s}, 2 \mathrm{H}, \mathrm{NH}_{2}\right), 3.67$ (q, 2H, $\left.\mathrm{CH}_{2}\right), 6.91-7.76(\mathrm{~m}, 9 \mathrm{H}, \mathrm{Ar}-\mathrm{H}$ and $\mathrm{NH})$. Found; C, 57.43; H, 3.67; N, 10.72; cald. For $\mathrm{C}_{25} \mathrm{H}_{19} \mathrm{ClN}_{4} \mathrm{O}_{3} \mathrm{~S}_{2}$ (523.04); C, 57.41; H, 3.66; N, 10.71 .

\section{Method (B)}

To a solution of $16 \mathrm{a}(0.01 \mathrm{~mole})$ in ethanol, ethylchloroacetate $(0.01$ mole $)$ and sodium ethoxide ( 0.01 mole sodium in $10 \mathrm{ml}$ ethanol) were added. The reaction mixture was refluxed for 12 hrs, then left to stand, poured into cold water (50 $\mathrm{ml}$ ) and acidified with $\mathrm{HCl}$. The product formed was collected by filteration and recrystallized from ethanol to give $(21 ; 77 \%)$ as yellow crystals. 
6-(1,3-Benzothiazol-2-ylamino)-4-methyl-2thioxo-1,2-dihydro-pyridine-3-carbonitrile (22):

A mixture of 1 (0.01 mole) and cyanothioacetamide (0.01 mole) in ethanol (30 $\mathrm{ml})$ was treated with a few drops of piperidine and refluxed for $8 \mathrm{hrs}$. The solid product was collected by filteration and recrystallized from ethanol to give $(22 ; 59 \%)$ as brown crystals; m.p. $190^{\circ} \mathrm{C}$; IR $(\mathrm{KBr}) \vee \mathrm{cm}^{-1}$ 3327, 3199 (2NH), 3059 (CHarom), 2928 (CH-aliph), $2203(\mathrm{C} \equiv \mathrm{N}) ;{ }^{1} \mathrm{H}$ NMR $\left(\mathrm{DMSO}_{6}\right) \delta=1.27\left(\mathrm{~s}, 3 \mathrm{H}, \mathrm{CH}_{3}\right), 7.27(\mathrm{~s}, 1 \mathrm{H}$, pyridine-H), 7.42-7.79 (m, 6H, Ar-H + 2NH). Found; C, 56.36; H, 3.39; N, 18.79; cald. For $\mathrm{C}_{14} \mathrm{H}_{10} \mathrm{~N}_{4} \mathrm{~S}_{2}$ (298.39); C, 56.35; H, 3.38; N, 18.78 .

Preparation of compounds (23a,b): General procedure

\section{$\operatorname{Method~(A)~}$}

A mixture of 1 (0.01 mole), appropriate active methylene reagent (malononitrile or cyanoacetamide) (0.01 mole) and ammonium acetate $(0.5 \mathrm{gm})$ was fused for 30 minutes at $140^{\circ} \mathrm{C}$. The reaction mixture was left to stand, then triturated with ethanol. The solid product so formed was collected by filteration and recrystallized from the proper solvent to give $23 \mathrm{a}, \mathrm{b}$.

\section{Method (B)}

A mixture of 1 (0.01 mole), appropriate active methylene reagent (malononitrile or cyanoacetamide) (0.01 mole) in ethanol $(30 \mathrm{ml})$ was treated with a few drops of piperidine and refluxed for $12 \mathrm{hrs}$, then left to cool. The solid product formed was filtered off and crystallized from the proper solvent to give 23a,b.

2-Amino-1-(1,3-benzothiazol-2-yl)-4-methyl-6oxo-1,6-dihydropyridine-3-carbonitrile (23a)

It was obtained as brown crystals from dioxane/ethanol; yield 74\%; m.p. $268-70^{\circ} \mathrm{C}$; IR $(\mathrm{KBr}) \vee \mathrm{cm}^{-1} 3350\left(\mathrm{NH}_{2}\right), 3090$ (CH-arom), $2200(\mathrm{C} \equiv \mathrm{N}), 1660(\mathrm{C}=\mathrm{O}) ;{ }^{1} \mathrm{H}$ NMR (DMSO-d $)$ $\delta=2.20\left(\mathrm{~s}, 3 \mathrm{H}, \mathrm{CH}_{3}\right), 5.72\left(\mathrm{~s}, 2 \mathrm{H}, \mathrm{NH}_{2}\right), 7.54-$ 8.19 (m, 5H, Ar-H + pyridine-H); MS: m/z = 282 $\left(\mathrm{M}^{+}\right)$. Found; C, 59.57; H, 3.58; N, 19.85; cald. For $\mathrm{C}_{14} \mathrm{H}_{10} \mathrm{~N}_{4} \mathrm{OS}$ (282.33); C, 59.56; H, 3.57; N, 19.84 .

2-Amino-1-(1,3-benzothiazol-2-yl)-4-methyl-6oxo-1,6-dihydropyridine-3-carboxamide (23b):

It was obtained as orang crystals from ethanol; yield $70 \%$; m.p. $180^{\circ} \mathrm{C}$; IR $(\mathrm{KBr}) \vee \mathrm{cm}^{-1} 3400$, $3350\left(\mathrm{NH}_{2}\right), 2930$ (CH-aliph), 1720, $1640(2 \mathrm{C}=\mathrm{O})$; ${ }^{1} \mathrm{H}$ NMR $\left(\mathrm{DMSO}_{6}\right) \delta=1.25\left(\mathrm{~s}, 3 \mathrm{H}, \mathrm{CH}_{3}\right), 5.23$ (s, 2H, $\mathrm{NH}_{2}$ ), 5.39 (hump, 2H, $\left.\mathrm{NH}_{2}\right), 6.17(\mathrm{~s}, 1 \mathrm{H}$, pyridine-H), 7.12-8.32 (m, 4H, Ar-H). Found; C, 55.98; H, 4.02; N, 18.64; cald. For $\mathrm{C}_{14} \mathrm{H}_{12} \mathrm{~N}_{4} \mathrm{O}_{2} \mathrm{~S}$ (300.34); C, 55.99; H, 4.03; N, 18.65.

Preparation of compounds (28a,b): General procedure

A mixture of acetoacetanilide 1 ( 0.01 mole), $\omega$-bromoacetophenone $(0.01$ mole $)$ and sodium hydride $(0.31 \mathrm{~g}, 0.01 \mathrm{~mole})$ in dry benzene (20 $\mathrm{ml}$ ) was stirred for $2 \mathrm{hr}$ at $40^{\circ} \mathrm{C}$. The separated solid was filtered off, treated with dil $\mathrm{HCl}$ (2:1), washed with excess of water and recrystallized from the proper solvent to give $(28 \mathrm{a}, \mathrm{b})$.

4-Methyl-3-[2-(4-Bromophenyl)-2-oxo-ethyl]2 H-pyrimido-[2,1-b][1,3]-benzothiazol-2-one (28a).

It was obtained as red crystals from ethanol; yield $67 \%$; m.p. $192-4^{\circ} \mathrm{C}$; IR $(\mathrm{KBr}) \vee \mathrm{cm}^{-1} 3080$ (CH-arom), 2980 (CH-aliph), $1669(\mathrm{C}=\mathrm{O}) ;{ }^{1} \mathrm{H}$ $\operatorname{NMR}\left(\mathrm{CDCl}_{3}\right) \delta=1.27\left(\mathrm{~s}, 3 \mathrm{H}, \mathrm{CH}_{3}\right), 3.71(\mathrm{~s}$, $\left.2 \mathrm{H}, \mathrm{CH}_{2}\right), 6.92-7.78$ (m, 8H, Ar-H). Found; C, 55.23; H, 3.19; N, 6.79; cald. For $\mathrm{C}_{19} \mathrm{H}_{13} \mathrm{BrN}_{2} \mathrm{O}_{2} \mathrm{~S}$ (413.30); C, 55.22; H, 3.17; N, 6.78.

4-Methyl-3-[2-(4-methylphenyl)-2-oxo-ethyl]2H-pyrimido-[2,1-b][1,3]-benzothiazol-2-one (28b)

It was obtained as brown crystals from ethanol; yield $77 \%$; m.p. $138-40^{\circ} \mathrm{C}$; IR (KBr) $v \mathrm{~cm}^{-1} 3040$ (CH-arom), 2922 (CH-aliph), $1680(\mathrm{C}=\mathrm{O}) ;{ }^{1} \mathrm{H}$ NMR (DMSO-d $\left.)_{6}\right) \delta=2.20\left(\mathrm{~s}, 3 \mathrm{H}, \mathrm{CH}_{3}\right), 2.43$ (s, $\left.3 \mathrm{H}, \mathrm{CH}_{3}\right), 3.37\left(\mathrm{~s}, 2 \mathrm{H}, \mathrm{CH}_{2}\right), 7.26-7.96(\mathrm{~m}, 8 \mathrm{H}$, Ar-H). Found; C, 68.96; H, 4.65; N, 8.06; cald. For $\mathrm{C}_{20} \mathrm{H}_{16} \mathrm{~N}_{2} \mathrm{O}_{2} \mathrm{~S}$ (348.43); C, 68.95; H, 4.63; N, 8.04 .

Preparation of compounds (30a,b): General procedure

To a solution of compound $\mathbf{1}(0.01$ mole $)$ in dry acetone $(50 \mathrm{ml})$, benzoyl or ethoxy carbonyl isothiocyanate $(0.01$ mole $)$ was added. The reaction mixture was heated under reflux for $12 \mathrm{hrs}$, then left to cool. The solid product was collected by filteration and recrystallized from the proper solvent to give $(30 \mathrm{a}, \mathrm{b})$.

5-Acetyl-3-(1,3-benzothiazol-2-yl)-2-phenyl-6thioxo-5, 6-dihydro-pyrimidin-4(3H)-one (30a):

It was obtained as pale yellow crystals from ethanol; yield $62 \%$; m.p. $190^{\circ} \mathrm{C}$; IR $(\mathrm{KBr}) \vee \mathrm{cm}^{-1}$ 3002 (CH-arom), 1713, $1668(2 \mathrm{C}=\mathrm{O}) ;{ }^{1} \mathrm{H}$ NMR $\left(\mathrm{DMSO}_{6}\right) \delta=2.14\left(\mathrm{~s}, 3 \mathrm{H}, \mathrm{COCH}_{3}\right), 7.29$ 7.89 (m, 9H, Ar-H), 7.97 (s, 1H, pyrimidin-H). Found; C, 60.15; H, 3.46; N, 11.08; cald. For 
$\mathrm{C}_{19} \mathrm{H}_{13} \mathrm{~N}_{3} \mathrm{O}_{2} \mathrm{~S}_{2}$ (379.46); C, 60.14; H, 3.45; N, 11.07 .

5-Acetyl-3-(1,3-benzothiazol-2-yl)-2-ethoxy-6thioxo-5,6-dihydropyrimidin-4 (3H)-one (30b):

It was obtained as brown crystals from Dioxane/EtOH; yield 71\%; m.p. 298-300 ${ }^{\circ} \mathrm{C}$; IR (KBr) $v \mathrm{~cm}^{-1} 3080$ (CH-arom), 2988 (CH-aliph), 1720, $1658(2 \mathrm{C}=\mathrm{O}) ;{ }^{1} \mathrm{H}$ NMR (DMSO-d $) \delta=$ $1.26\left(\mathrm{t}, 3 \mathrm{H}, \mathrm{CH}_{3}\right), 2.06\left(\mathrm{~s}, 3 \mathrm{H}, \mathrm{COCH}_{3}\right), 4.23(\mathrm{q}$, $\left.2 \mathrm{H}, \mathrm{CH}_{2}\right), 7.25-8.01(\mathrm{~m}, 5 \mathrm{H}, \mathrm{Ar}-\mathrm{H}+$ pyrimidin- $\mathrm{H})$. Found; C, 51.85; H, 3.76; N, 12.08; cald. For $\mathrm{C}_{15} \mathrm{H}_{13} \mathrm{~N}_{3} \mathrm{O}_{3} \mathrm{~S}_{2}$ (347.42); C, 51.86; H, 3.77; N, 12.09 .

5-Methyl-2-phenyl-pyrazolo[1,5-a]pyrimidin-7one (33)

A mixture of 1 (0.01 mole) and (0.01 mole) of aminopyrazole derivatives in benzene (30 $\mathrm{ml})$ containing glacial acetic acid $(10 \mathrm{ml})$ was heated under reflux for $3 \mathrm{hrs}$. The solvent was then evaporated and the resulting solid product was filtered off and recrystallized from DMF/ EtOH to give $(33 ; 69 \%)$ as colorless crystals; m.p. $>300^{\circ} \mathrm{C}$; IR $(\mathrm{KBr}) \vee \mathrm{cm}^{-1} 3534(\mathrm{NH}), 3060(\mathrm{CH}-$ arom), 2892 (CH-aliph), $1662(\mathrm{C}=\mathrm{O}) ;{ }^{1} \mathrm{H}$ NMR $\left(\mathrm{DMSO}_{\mathrm{d}}\right) \delta=2.31\left(\mathrm{~s}, 3 \mathrm{H}, \mathrm{CH}_{3}\right), 5.61(\mathrm{~s}, 1 \mathrm{H}$, pyrazole-H), $6.58(\mathrm{~s}, 1 \mathrm{H}$, pyrimidine- $\mathrm{H}), 7.40-$ 8.00 (m, 5H, Ar-H), 12.57 (hump, 1H, NH); MS: $\mathrm{m} / \mathrm{z}=225\left(\mathrm{M}^{+}\right)$. Found; $\mathrm{C}, 69.34 ; \mathrm{H}, 4.93 ; \mathrm{N}$, 18.66; cald. For $\mathrm{C}_{13} \mathrm{H}_{11} \mathrm{~N}_{3} \mathrm{O}$ (225.25); C, 69.32; H, $4.92 ; \mathrm{N}, 18.65$.

Preparation of compounds (37a,b): General procedure

A cold solution of diazonium aminopyrazole salt $(10 \mathrm{mmol})$ (perpared by adding a solution of sodium nitrite ( $1.5 \mathrm{gm}$ into $\left.10 \mathrm{ml} \mathrm{H}_{2} \mathrm{O}\right)$ to cold solution of amine hydrochloride $(0.1$ mole in 10 $\mathrm{ml}$ concentrated $\mathrm{HCl}$ ) was stirring in ice bath. The resulting solution of diazonium salt was then added to cold solution of compound 1 ( 0.01 mole $)$ in ethanol $(30 \mathrm{ml})$ containing $2 \mathrm{gm}$ of sodium acetate at $0^{\circ} \mathrm{C}$ for $1 \mathrm{hr}$. The resulting solid was washed with water and recrystallized from the proper solvent to give $37 \mathrm{a}$,b.

7-Amino-4-methyl-8-[4-(thiazol-2-yl-sulfamoyl)phenylazo]-pyrazolo [5,1-c][1,2,4]triazine-(1,3benzothiazol-2-yl)-3-carboxamide (37a)

It was obtained as brown crystals from ethanol; yield $77 \%$; m.p. $186^{\circ} \mathrm{C}$; IR $(\mathrm{KBr}) \vee \mathrm{cm}^{-1} 3300$, $3280\left(\mathrm{NH}_{2}\right), 3220,3146(2 \mathrm{NH}), 3020$ (CH-arom), $1659(\mathrm{C}=\mathrm{O}) ;{ }^{1} \mathrm{H}$ NMR $\left(\right.$ DMSO-d $\left._{6}\right) \delta=2.22(\mathrm{~s}$, $\left.3 \mathrm{H}, \mathrm{CH}_{3}\right), 6.82-8.05(\mathrm{~m}, 12 \mathrm{H}, \mathrm{Ar}-\mathrm{H}$, thiazole- $\mathrm{H}$ and $\mathrm{NH}_{2}$ ), 12.72 (hump, $\left.1 \mathrm{H}, \mathrm{NH}\right), 13.21(\mathrm{~s}, 1 \mathrm{H}$, Egypt.J.Chem. 61, No.6 (2018)
NH). Found; C, 46.70; H, 2.92; N, 26.05; cald. For $\mathrm{C}_{23} \mathrm{H}_{17} \mathrm{~N}_{11} \mathrm{O}_{3} \mathrm{~S}_{3}(591.66) ; \mathrm{C}, 46.69 ; \mathrm{H}, 2.90 ; \mathrm{N}$, 26.04 .

4-Methyl-7-oxo-8-[4-(guanidin-2-yl-sulfamoyl)phenylazo]-pyrazolo $[5,1-c][1,2,4]$ triazine- $(1,3-$ benzothiazol-2-yl)-3-carboxamide (37b)

It was obtained as brown crystals from ethanol; yield $71 \%$; m.p. $270^{\circ} \mathrm{C}$; IR $(\mathrm{KBr}) \vee \mathrm{cm}^{-1} 3444$, $3425\left(\mathrm{NH}_{2}\right), 3329,3220(2 \mathrm{NH}), 2980$ (CH-aliph), $1636(\mathrm{C}=\mathrm{O}) ;{ }^{1} \mathrm{H}$ NMR $($ DMSO-d $) ~ \delta=2.48(\mathrm{~s}$, $3 \mathrm{H}, \mathrm{CH}_{3}$ ), 6.70 (hump, $2 \mathrm{H}, \mathrm{NH}_{2}$ ), 7.34-8.05 (m, $10 \mathrm{H}, \mathrm{Ar}-\mathrm{H}$ and $2 \mathrm{NH}), 12.65$ (s, 1H, NH), 13.26 (s, 1H, NH). Found; C, 45.74; H, 3.13; N, 27.95; cald. For $\mathrm{C}_{21} \mathrm{H}_{17} \mathrm{~N}_{11} \mathrm{O}_{4} \mathrm{~S}_{2}$ (551.57); C, 45.73; H, $3.11 ; \mathrm{N}, 27.93$.

\section{References}

1. Musser J. H., Brown R. E., Loev B., Bailey K., Jones H., Kahen R., Fu-Chih Huang, Khandwala A., Leibowitz M., Sonnino-Goldman P. and Ruzza D. D., J. Med. Chem., 27, 121 (1984).

2. Russo F. and Santagati M., Farmaco. Ed. Sci., 31, 41 (1976).

3. Ghoneim K. M., El-Basil S., Osman A. N., Said M. M. and Megahed S. A., Rev. Roum. Chim., 36, 1355 (1991).

4. Singh S. P. and Segal S., Indian J. Chem., 27B, 941 (1988).

5. Sawhney S. N. and Bansal O. P., Indian J. Chem., 15B, 121 (1977)

6. Brown H. D., US Pat., 3, 278, 547, (1966), Chem. Abstr., 65, 18593 (1966).

7. Takeda Chemical Industries, Ltd.; Jpn.Kokai Tokkyo Toho Jpn., 59, 36, (1984), 670, Chem. Abstr., 101, 130683q (1984).

8. Hagen H. and Kohler R. D.: Ger. Pat., 2, 950, 291, (1981), Chem. Abstr., 95, 187261 (1981).

9. Papenfuh T. : Ger: Pat., 3, 528, 230, (1987), Chem. Abstr., 106, 213936 (1987)

10. Kendall J. D., Waddington R. H. J., Duffin G. F. : Brit, Pat., 867, 592, (1961), Chem. Abstr., 55, 21927 (1961).

11. Hussein A. M. , Z. Naturforsch, 53b, 1 (1998).

12. Hussein A. M. , Shrief S. M. and Atalla A. A., Monatshefte Fur Chemie, 127, 1153 (1996). 
13. Rathod A. S. S., Berad B. N., Patil S. D. and Doshi A. G., Orient. J. Chem., 16 (2), 315-318 (2000).

14. Ojha K. G., Neera J. and Heer T., Journal of the Indian Chemical Society, 79 (2), 191-192 (2002).

15. Gelbard G., Lin J. and Roques N., J. Org. Chem., 57, 1789-1793 (1992).

16. Folkers K., Harwood H.J. and Johnson T.B., J. Am. Chem. Soc., 54, 3751 (1932).

17. Del. Corona L., Signrelli G., Pinzetta A. and Coppi G., Eur. J. Med. Chem., 27, 419-423 (1992).
18. Al-Mousawi, S., Elassar, A-Z., El-Apasery, M.A., Phosphorus. Sul. Sil. Rel. Elem., 181, 17551771(2006).

19. El-Apasery, M.A., Pol. J. Appl. Chem., 50, 7581(2006).

(Received 24/5/2018; accepted 18/7/2018)

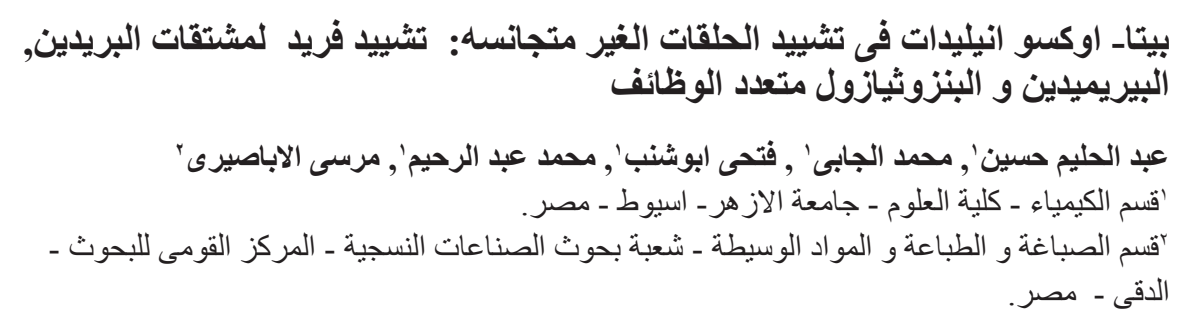

تم تشييد فريد لمشتقات البريدين , البيريميدين و البنزوثيازول متعدد الوظائف و على سبيل المثال مفاعلة

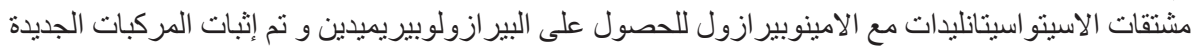

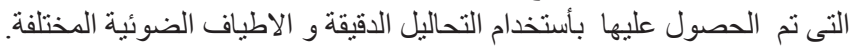

